\title{
28 Research Square \\ Characteristics of immune cell infiltration landscape in colorectal cancer to Aid in Immunotherapy
}

\section{Fei Kuang}

Changhai Hospital of the Second Military Medical University

De Luo

Affiliated Hospital of Southwest Medical University

\section{Mengjia Zhou}

Seventh People's Hospital of Shanghai University of Traditional Chinese Medicine

\section{Juan Du}

Southwest Medical University

\section{Yuee Liu}

Changhai Hospital of the Second Military Medical University

\section{Yuehua Li ( $\square$ liyuehua2020@stu.usc.edu.cn)}

University of South China

\section{Research Article}

Keywords: immune cell infiltration, the tumor microenvironment, prognostic biomarker, colorectal cancer, score

Posted Date: May 5th, 2021

DOI: https://doi.org/10.21203/rs.3.rs-467215/v1

License: (c) (i) This work is licensed under a Creative Commons Attribution 4.0 International License.

Read Full License 


\section{Abstract}

The tumor microenvironment (TME) is a complex environment composed of a variety of stromal cells and immune cells that infiltrate the tumor space. Recent clinical work has clearly shown such intratumoral immune cell infiltration $(\mathrm{ICl})$ to be closely related to colorectal cancer (CRC) patient survival, yet the specific landscape of infiltrating immune cells associated with this cancer type remains to be clarified. We utilized two computational algorithms to evaluate the ICl status of $712 \mathrm{CRC}$ patients, stratifying these patients into two ICl status-based patterns and assigning $\mathrm{ICl}$ scores through the use of principal component analyses. We found that the overall survival (OS) of patients with higher ICI scores was significantly longer than that of patients with low ICl scores. When ICI scores were combined with the results of tumor mutational burden (TMB) analyses, we determined that CRC patients with both high ICI scores and low TMB exhibited the best survival outcomes. High expression of MARCO in those patients with low ICl scores was correlated with reduced natural killer (NK) and effector T cell infiltration and with increased regulatory $T$ cell infiltration, suggesting that these factors may be linked to poor patient prognosis. These results suggest $\mathrm{ICl}$ scores to be a valuable biomarker for the prognostic evaluation of CRC patients. Future efforts to analyze the ICl patterns of larger patient sample cohorts will help to extend these analyses, offering new insights into the role of the TME in cancer progression while highlighting novel immunotherapeutic approaches to treating this cancer type.

\section{Introduction}

Colorectal cancer (CRC) is among the most common and deadliest cancers in developed nations, with an estimated 400,000 CRC diagnoses and 212,000 deaths globally each year [1]. Immunotherapeutic treatments serve to augment the ability of natural host defense mechanisms to recognize and remove tumor cells, and such regimens have proven effective in synergistically enhancing the survival of patients with a range of tumor types [2-4]. However, immunotherapies generally only benefit a small percentage of patients, and there is thus a clear need to better understand which therapeutic markers can be analyzed to gauge CRC patient responses to immunotherapeutic treatment [2-5].

The tumor microenvironment (TME) is a complex setting that influences cancer development and progression [6]. The TME contains a diverse array of stromal cells, lymphatic structures, and blood vessels, all of which can influence and be influenced by the oncogenic mutations driving the onset and evolution of a given cancer. The infiltration of various immune cell types into the TME has been shown to be an effective prognostic biomarker for tumor invasion status, offering insights into cancer grade, stage, and metastasis [7-8]. Tumor-associated macrophages (TAMs), for example, can produce cytokines including interleukin-10 (IL-10) and transforming growth factor-B (TGF-B) that can suppress immune cell activation, thereby promoting tumor proliferation and contributing to poorer patient prognosis [9-11]. In contrast, higher levels of tumor-infiltrating lymphocytes (TILs) including both CD $4+$ and CD8 + T cells are related to improved patient survival and immunotherapeutic responsiveness [12]. 
Immune checkpoint blockade therapies rely on the activation of extant TILs, enhancing their ability to recognize tumor cells and to thereby engage appropriate immunotherapeutic responses [13-14]. Merely identifying these TILs, however, is not sufficient to reliably characterize the complexities of the TME, as many patients with high TIL levels nonetheless exhibit resistance to immunotherapy treatment [15-16]. Such effects may be in part attributable to TAM-derived immunosuppressive cytokine production, or to stromal infiltration of the tumor, as this can interfere with TIL accumulation [14-15]. These prior results suggest that dynamic interactions between cell types are more important in the TME than are any individual cell population from a prognostic perspective. Prior studies have not performed a high-level analysis of the prognostic relevance of different immune cell infiltration (ICI) populations in CRC.

Herein, we employed the CIBERSORT and ESTIMATE algorithms to characterize CRC patient tumor gene expression profiles in an effort to comprehensively characterize intratumor immune cell landscape characteristics within these tumors [17-18]. We then stratified CRC patient tumors into two subtypes based upon the observed degree of $\mathrm{ICl}$, and we established $\mathrm{ICl}$ scores that were successfully used to gauge patient prognosis and which may offer value for the design of novel immunotherapeutic regimens.

\section{Materials And Methods}

\section{Dataset selection}

All data used in the present study were derived from The Cancer Genome Atlas (TCGA) (https://portal.gdc.cancer.gov/) and Gene Expression Omnibus (GEO) (https://www.ncbi.nlm.nih. gov/geo/) databases under the accession number GSE17538 (Fig.1A).

\section{Tumor-Infiltrating Immune Cell Consensus Clustering}

The CIBERSORT R package 21 was used to estimate tumor infiltration by different immune cell populations based upon 1000 permutations and LM22 markers, with both immune cell and matrix contents being evaluated to yield specific immune and matrix scores. CRC patient samples were then clustered according to sample-specific ICI patterns. A PAM-based approach relying upon Euclidean and Ward's linkages was utilized for unsupervised clustering with the ConsensuClusterPlus $\mathrm{R}$ package, and clustering was conducted 1000 times to ensure stable classification.

\section{Identification of ICI-related gene expression patterns}

ICI profiles were used to group CRC patients into different ICI clusters, after which differentially expressed genes (DEGs) between these clusters were compared with the following cutoff criteria using the limma R package: adjusted $\mathrm{p}<0.05$; absolute fold-change $>1.4$.

\section{Dimensional Reduction and ICI Score Calculation}

An unsupervised clustering approach was initially used to classify patients according to DEG expression values, with DEGs that were positively and negatively correlated with key clustering-related features being respectively referred to as ICI gene features A and B. Dimensional reduction was then performed for these ICI-related genetic signatures using a Boruta algorithm, and principal component 1 in the resultant principal component analysis (PCA) was then extracted as a signature score. A method similar to that previously used to compute a gene expression grade index [42] was then employed to calculate individual patient ICI scores as follows: 


\section{ICI score $=\sum P C 1_{A}-\sum P C 1_{B}$}

\section{Somatic Alteration Data Compilation}

Mutational data corresponding to CRC patients in the TCGA database was downloaded and used to estimate tumor mutational burden (TMB). CRC driver genes were identified with the maftool R package, and somatic mutations therein were evaluated for those with high and low ICI scores. The top 20 most frequently mutated driver genes were then retained for further analysis.

\section{Statistical Analyses}

GraphPad Prism 7.0 and SPSS 21.0 (IBM, NY, USA) were used for all statistical testing. Data between groups were compared via the Wilcoxon test. Patient classification into two ICI subtypes was achieved with the X-tile software via an iterative approach aimed at reducing computational batch effects [43]. Kaplan-Meier plotter was employed to generate all survival curves, which were analyzed with log-rank tests. Chi-squared tests were employed to assess correlations between ICI score subgroups and somatic mutation frequencies, while correlations were assessed via Spearman's correlation analyses. A two-tailed $\mathrm{p}<0.05$ was the significance threshold for this study.

\section{Results}

\section{Assessment of the CRC-related TME Immune Cell Infiltration Landscape}

We began by employing the ESTIMATE and CIBERSORT algorithms to gauge the relative enrichment of different immune cell populations in samples of tumors from 712 CRC patients (Fig. 1A). Data were obtained from the TCGA and GEO databases (Accession number: GSE17538), and analyzed tumor cells exhibited matched immune cell infiltration (ICI) profiles. The ConsesusClusterPlus R package was then used to classify these CRC patients into distinct ICl-related subtypes via an unsupervised clustering approach (Fig. 1B).

Significant differences in overall survival (OS) were evident between patients with the two independent ICl-related CRC subtypes identified in this study ( $p=0.047$; Fig. 1C). To better understand the biological basis for these prognostic relationships, we further assessed the immune cell makeup of the TME in these patient clusters (Clusters A and B). Patients in cluster A had a better prognosis, and exhibited tumors predicted to contain higher levels of naive B cells, M1 macrophages, memory CD4 T cells, CD8 T cells, NK cells, monocytes, and plasma cells. Patients in ICl cluster B had a median survival of 7 years, and exhibited tumors predicted to contain higher levels of regulatory T cells (Tregs), M0 macrophages, and activated mast cells (Fig. 1D-E). Correlation coefficient heatmaps were also prepared to visualize the immune cell infiltration landscape of the TME in these CRC patient cohorts (Fig. 1F).

We further assessed the relative expression of the key immune-related gene MARCO in these two ICI patient clusters, revealings significantly increased MARCO expression among patients in cluster B relative to those in cluster A. Differences between immune cell types and MARCO expression levels in these patient cohorts were then evaluated (Fig. 1G). 


\section{Immune Gene-Related Subtype Analyses}

To better understand the biological basis for the ICI subtypes of CRC patients identified above, we conducted a differential transcriptomic analysis of these patient cohorts with the $\mathrm{R}$ limma package. Through unsupervised clustering of 34 identified DEGs, these patients were stratified into two genomic clusters (clusters A and B) (Fig. 2A). In total, 23 of these DEGs were positively correlated with this gene cluster and were denoted as ' $\mathrm{ICl}$ gene signature $A$ ', whereas the remaining DEGs were referred to as ' $\mathrm{ICl}$ gene signature $\mathrm{B}^{\prime}$. The Boruta algorithm was then used to conduct a dimensionality reduction for these two ICl gene signatures in order to reduce computational noise associated with these analyses [19]. The clusterProfiler $\mathrm{R}$ package was then used to prepare a heatmap separating the transcriptomic profiles of these 34 DEGs across genomic clusters (Fig. 2B) as described previously [20], with significantly enriched biological processes associated with these genes being shown in Figs. $2 \mathrm{C}$ and 2D.

The prognostic relevance of these $\mathrm{ICl}$ gene clusters was next evaluated using the Kaplan-Meier plotter tool, revealing that patients in cluster $A$ had a better prognosis relative to patients in cluster $B(p=0.002$; Fig. 2E). Significant differences in MARCO gene expression were also detected between these two genomic clusters $(p<0.001$; Fig. $2 F)$, with the expression of this key immunological gene being significantly higher in samples from patients in cluster B relative to those from patients in cluster $A$.

\section{Evaluating Correlations between ICI Scores and Somatic Variation}

A PCA approach was next employed to compute aggregate ICl scores for the two ICl gene signatures detailed above in an effort to quantitatively evaluate the ICI landscape of CRC. The resultant scores (ISA and ISB for genes in signatures A and B, respectively) were then calculated for each patient by summing the relevant individual scores, yielding a prognostic signature which was defined as an $\mathrm{ICl}$ score. The patients in our study cohort were then separated into two groups based upon whether they had high or low ICl scores, with the cutoff value used to differentiate these two groups being established with the Xtile software. Patient distributions in these two gene clusters are shown in Fig. 3A. We then evaluated immune activity in each patient group before assessing the prognostic relevance of our ICl scores, revealing that most immune checkpoint- and immune activity-related genes were significantly overexpressed in samples from the low ICI group with the exception of PIGR, IGLJ3, CLCA1, ITLN1, IGHM, PLA2G2A, CLCA4, ZG16, UGT2B17, REG4, and SPINK4 (Fig. 3B). TAMs expressing MARCO can interfere with the activation of NK and T cells, suppressing their cytokine production, proliferation, and cytotoxicity, while simultaneously aiding Treg proliferation (21). In line with such a model, we found that high MARCO expression in individuals with low $\mathrm{ICl}$ score subtypes was correlated with higher Treg infiltration and reduced NK and effector T cell levels, potentially explaining the poorer prognosis for these patients. A gene set enrichment analysis (GSEA) further showed the regulation and vascular signaling pathways to be significantly enriched among patients in the low $\mathrm{ICl}$ score group, while the butanoate and retinol signaling pathways were significantly enriched among those in the high ICl group (Figs. 3C). 
Many prior studies have shown that tumors bearing a high mutational burden are more likely to exhibit increased $\mathrm{CD} 8^{+} \mathrm{T}$ cell infiltration, aiding in their elimination. As such, TMB may predict a given cancer patient's responsiveness to immunotherapy treatment [22-23]. We therefore evaluated potential relationships between $\mathrm{TMB}$ and $\mathrm{ICl}$ scores in $\mathrm{CRC}$ pateints, evaluating somatic variant distributions in $\mathrm{CRC}$ driver genes in the low and high $\mathrm{ICl}$ score patient subgroups using maftools [24]. We then retained the top 20 driver genes with the highest mutational frequency for further analysis (Fig. 3D-3E).

\section{Assessment of the Prognostic Value of ICI Scores}

Finally, we conducted an in-depth analysis of the prognostic relevance of the ICl scores computed above using the Kaplan-Meier plotter tool. The OS of patients in the high ICI score group was significantly better than that of those in the low $\mathrm{ICl}$ score group $(p=0.004 ; \mathrm{Fig}$. $4 \mathrm{~A})$. We then evaluated the synergistic value of $\mathrm{ICl}$ and TMB scores as predictors of CRC patient survival in a stratified survival analysis which found TMB status to be unrelated to ICI score-based prognostic analyses such that higher ICI scores were linked to better patient $\mathrm{OS}$ regardless of TMB status (High TMB + High ICl score $(\mathrm{HH})$ vs. High TMB + Low ICI score (HL), Low TMB + high ICl score (LH) vs. Low TMB + Low ICl score (LL), $p=0.006$; Fig. 4B). Together these data suggested that $\mathrm{ICl}$ scores serve as prognostic biomarkers that are independent of TMB status. The mortality rate for patients with high $\mathrm{ICl}$ scores was also decreased relative to that of patients with low $\mathrm{ICl}$ scores (Fig. 4C), and the ICl scores of surviving patients were significantly higher than those of nonsurviving patients $(p=0.027$; Fig. 4D)

We additionally evaluated the relationship between the prognostic value of $\mathrm{ICl}$ scores and other clinically relevant parameters in $\mathrm{CRC}$ patients. This approach revealed high $\mathrm{ICl}$ scores to be associated with significantly better survival among individuals $\leq 65$-years-old $(p=0.024$; Fig. $4 \mathrm{E})$ and those with advanced CRC ( $p=0.01$; Fig. $4 F)$,. This also remained true regardless of patient gender (male $p=0.04$; female $p=0.026$; Fig. 4G-4H).

\section{Discussion}

Immunotherapeutic treatment represents a highly efficacious approach to controlling the growth of many tumors, and can improve the quality of life for advanced CRC patients. However, many patients fail to respond to immunotherapies, and the Association for Cancer Immunotherapy has emphasized the importance of identifying those patients most likely to benefit from these therapeutic regimens [25].

Herein, we developed a quantitative approach to evaluating the immunological TME associated with CRC patient tumors. The results of this study indicate that $\mathrm{ICl}$ scores represent a valuable prognostic biomarker that can be used to predict outcomes in these patients, and we found that higher ICl scores as well as increased intratumoral CD4+, CD8+, plasma cell, and macrophage infiltration were linkedto better patient outcomes, in line with prior research [26-27]. This underscores the potential for already extant immune responses to achieve anti-tumor effects and to thereby affect the way cancer patients respond to immunotherapy. CRC tumors are thought to exhibit among the highest levels of immune cell infiltration on average [28]. We thus hypothesized that comprehensively characterizing ICI profiles and related 
patterns of gene expression would represent a novel approach to developing patient-specific evaluation and treatment strategies. We began by characterizing the TME associated with CRC tumors at a molecular level, enabling us to identify immune-related genes based upon ICl gene clusters. ICI gene cluster B was associated with lower immune scores, matrix scores, and with decreased immune cell infiltration consistent with what is often referred to as a 'cold' immune phenotype. In contrast, ICl gene cluster A was associated with higher immune scores and inflammatory cell infiltration, and these patients had a more favorable prognosis as well as increased CD $8+T$ cell, activated CD $4+T$ cell, and plasma cell infiltration [29-30]. Indeed, many prior studies have highlighted the effects of the TME on cancer patient OS [31]. The anti-tumor immune responses observed for patients in ICl gene cluster A suggest that they are likely to attain more benefits from immunotherapeutic treatment, and these clusters may thus offer value for the development of more efficacious immunotherapies. However, given the significant heterogeneity associated with the immunological TME in a given patient, it is vital that ICI patterns be evaluated on a patient-by-patient basis. Consistent with such an approach, one research group has reported to use of an individually-tailored tumor-specific biomarker model that can more reliably gauge breast cancer patient prognosis [32].

Using the Boruta algorithm, we established an $\mathrm{ICl}$ score that we were then able to analyze as a biomarker of CRC patient prognosis. GSEA analyses of these genes composing these ICl-related patterns revealed the regulation and vascular signaling pathways to be significantly enriched among samples from those with low $\mathrm{ICl}$ scores, while butanoate and retinol signaling pathways were enriched among those with high $\mathrm{ICl}$ scores. Recent wor has clarified the relationship between genetic mutations and patient sensitivity to immunotherapy [33-34]. By leveraging this fact and evaluating key immunological parameters including consensus ICl scores, tumor driver mutation analyses [35], TMB measurements [36], LOH HLA (loss of heterozygosity at the HLA locus) assessments [37-38], PD-L1 expression analyses [39], and the detection of key immune gene expression-related signatures [40-41], it is possible to more reliably classify particular cancers. Integrated ICl scores offered new insights regarding differences in variant frequencies in many genes when comparing samples from the low and high ICl score groups, with some of these genes being directly linked to therapeutic sensitivity or resistance. When we performed a stratified analysis, we determined the prognostic value of $\mathrm{ICl}$ scores to be independent of TMB status for CRC patients, suggesting that these two metrics offer distinct insights into the immunobiology of patient tumors, enabling the more robust assessment of patient outcomes. After employing two different algorithms to classify 712 CRC patients based upon their ICl profiles, we identified two ICl patterns and were able to employ a PCA approach to derive ICI scores therefrom. Higher ICl scores were associated with significantly higher patient OS relative to lower ICl scores, and this remained true in patients with advanced disease and in those $\leq 65$-years-old. High MARCO expression in those with low ICI score subtype disease was correlated with increased Treg infiltration and reduced levels of NK and effector T cells, which may be linked to poorer patient prognosis.

Lastly, we explored ICI scores and associated patient characteristics, and found that simultaneous analyses of ICl scores and TMB status may improve our ability to reliably gauge CRC patient prognosis, offering a means of potentially identifying patients at a higher risk of tumor recurrence, although further 
research is required to test this possibility. ICl scores nonetheless represent a powerful tool for estimating tumor-specific immune fitness, and can be used to predict which patients are most likely to benefit from immunotherapy, thereby helping to improve CRC patient survival.

\section{Conclusions}

Overall, we found that ICI scores can be reliably leveraged to assess CRC patient prognosis, with higher $\mathrm{ICI}$ scores being associated with prolonged OS. Stratified analyses revealed the prognostic value of ICI scores to be independent of TMB status for this cancer type, and this, together with observed GSEA and predictive outcomes, suggests that TMB and ICl status are independent tumor characteristics that may predict patient responses to immunotherapeutic treatment. We further found that that high MARCO expression in CRC patients with low ICl scores was correlated with reduced NK and effector T cell infiltration and increased Treg infiltration, potentially thereby contributing to poorer patient outcomes. Future studies of larger CRC patient cohorts will continue to offer new insights into the role of the TME as a driver of cancer progression, and may aid in the design of novel immunotherapeutic approaches to CRC treatment.

\section{Abbreviations}

TME

the tumor microenvironment

CRC

colorectal cancer

$\mathrm{ICl}$

immune cell infiltration

NK Cells

Natural Killer cells

TMB

tumor mutation burden

TAMs

tumor-associated macrophages

TLSs

tumor-infiltrating lymphocytes

TCGA

The Genomic Data Commons Data Portal

GEO

Gene Expression Omnibus

PCA

principal component analysis

DEGs 
differentially expressed genes

GSEA

gene set enrichment analysis

OS

Overall survival

Tregs

T cells regulatory

\section{Declarations}

\section{Availability of data and materials}

All data generated or analyzed during this study are included in this published article.

\section{Conflicts of Interest}

The authors declare there is no conflict of interest.

\section{Funding Statement}

This study was supported by the grant from the National Natural Science Foundation of China (NO. 81902707)

\section{Contributions}

F.K conceived and designed the whole project. D.L and M.J.Z analyzed the data and wrote the manuscript. J.D carried out data interpretations and data analysis. Y.H.L and Y.E.L revised the manuscript. All authors read and approved the final manuscript.

\section{Acknowledgements}

Not applicable.

\section{Ethics approval and consent to participate}

Not applicable.

\section{Consent for publication}

Consent for publication was obtained from all key informants who took place in the study.

\section{References}

1. Ferlay, J. et al. Estimates of worldwide burden of cancer in 2008: GLOBOCAN 2008. Int J Cancer. 127 (12), 2893-2917 (2010). 
2. Curran, M. A. et al. PD-1 and CTLA-4 combination blockade expands infiltrating T cells and reduces regulatory T and myeloid cells within B16 melanoma tumors. Proc Natl Acad Sci USA. 107 (9), 42754280 (2010).

3. Garassino, M. C. et al. Patient-reported outcomes following pembrolizumab or placebo plus pemetrexed and platinum in patients with previously untreated, metastatic, non-squamous nonsmall-cell lung cancer (KEYNOTE-189): a multicentre, double-blind, randomised, placebo-controlled, phase 3 trial. Lancet Oncol. 21 (3), 387-397 (2020).

4. Robert, C. et al. Anti-programmed-death-re-ceptor-1 treatment with pembrolizumab in ipilimumabrefractory advanced melanoma: a randomised dose-comparison cohort of a phase 1 trial. Lancet. 384 (9948), 1109-1117 (2014).

5. Hamid, O. et al. Safety and tumor responses with lambrolizumab (anti-PD-1) in melanoma. N Engl J Med. 369 (2), 134-144 (2013).

6. Nyamundanda, G., Fontana, E. \& Sadanandam, A. Is the tumour microenvironment a critical prognostic factor in early-stage colorectal cancer? Ann Oncol. 30 (10), 1538-1540 (2019).

7. Galon, J. et al. Type, density, and location of immune cells within human colorectal tumors predict clinical outcome. Science. 313 (5795), 1960-1964 (2006).

8. Galon, J., Fridman, W. H. \& Pages, F. The adaptive immunologic microenvironment in colorectal cancer: a novel perspective. Cancer Res. 67 (5), 1883-1886 (2007).

9. Noy, R. \& Pollard, J. W. Tumor-associated macrophages: from mechanisms to therapy. Immunity. 41 (1), 49-61 (2014).

10. De Palma, M. \& Lewis, C. E. Macrophage regulation of tumor responses to anticancer therapies. Cancer Cell. 23 (3), 277-286 (2013).

11. Chen, Y. P. et al. Identification and validation of novel microenvironment-based immune molecular subgroups of head and neck squamous cell carcinoma: implications for immunotherapy. Ann Oncol. 30 (1), 68-75 (2019).

12. Vassilakopoulou, M. et al. Evaluation of PD-L1 Expression and Associated Tumor-Infiltrating Lymphocytes in Laryngeal Squamous Cell Carcinoma. Clin. Cancer Res. 22 (3), 704-713 (2016).

13. Rosenberg, J. E. et al. Atezolizumab in patients with locally advanced and metastatic urothelial carcinoma who have progressed following treatment with platinum-based chemotherapy: a singlearm, multicentre, phase 2 trial. Lancet. 387 (10031), 1909-1920 (2016).

14. Chen, D. S. \& Mellman, I. Elements of cancer immunity and the cancer-immune set point. Nature. 541 (7637), 321-330 (2017).

15. xSenbabaoglu, Y. et al. Tumor immune microenvironment characterization in clear cell renal cell carcinoma identifies prognostic and immune therapeutically relevant messenger RNA signatures. Genome Biol. 17 (1), 231 (2016).

16. Ayers, M. et al. IFN-g-related mRNA profile predicts clinical response to PD-1 blockade. J Clin Invest. 127 (8), 2930-2940 (2017). 
17. Yoshihara, K. et al. Inferring tumour purity and stromal and immune cell admixture from expression data. Nat Commun. 4, 2612 (2013).

18. Newman, A. M. et al. Robust enumeration of cell subsets from tissue expression profiles. Nat Methods. 12 (5), 453-457 (2015).

19. Kursa, M. B. \& Rudnicki, W. R. Feature Selection with the Boruta Package. J Stat Softw. 36 (11), 1-13 (2010).

20. Yu, G., Wang, L. G. \& Han, Y. at el. clusterProfiler: an R package for comparing biological themes among gene clusters. OMICS 2012; 16(5):284-7.

21. Linnéa, L. et al. Targeting MARCO and IL-37R on immunosuppressive macrophages in lung cancer blocks regulatory T cells and supports cytotoxic lymphocyte function.Cancer Research.2020:1885. DOI: $10.1158 / 0008-5472$

22. Rizvi, N. A. et al. Cancer immunology. Mutational land-scape determines sensitivity to PD-1 blockade in non-small cell lung cancer. Science. 348 (6230), 124-128 (2015).

23. McGranahan, N. et al. Clonal neo-antigens elicit T cell immunoreactivity and sensitivity to immune checkpoint blockade. Science. 351 (6280), 1463-1469 (2016).

24. Mayakonda, A. et al. Maftools: efficient and comprehensive analysis of somatic variants in cancer. Genome Res. 28 (11), 1747-1756 (2018).

25. Ottaiano, A. et al. Evolution of Mutational Landscape and Tumor Immune-Microenvironment in Liver Oligo-Metastatic Colorectal Cancer. Cancers. 12 (10), 3073 (2020).

26. Zhou, R. et al. Immune cell infiltration as a biomarker for the diagnosis and prognosis of stage I-III colon cancer. Springer Open Choice. 68 (3), 433-442 (2019).

27. Rooney, M. S. et al. Molecular and genetic properties of tumors associated with local immune cytolytic activity. Cell. 160 (1-2), 48-61 (2015).

28. Angell, H. K. et al. The Immunoscore: Colon Cancer and Beyond. Clin Cancer Res. 26 (2), 332-339 (2020).

29. Hamanishi, J. et al. Programmed cell death 1 ligand 1 and tumor-infiltrating CD $8+T$ lymphocytes are prognostic factors of hu-man ovarian cancer. Proc Natl Acad Sci USA. 104 (9), 3360-3365 (2007).

30. Hwang, M. L., Lukens, J. R. \& Bullock, T. N. Cognate memory CD 4 + T cells generated with dendritic cell priming influence the expansion, trafficking, and differentiation of secondary CD $8+T$ cells and enhance tumor control. J Immunol. 179 (9), 5829-5838 (2007).

31. Li, B., Cui, Y. \& Nambiar, D. K. The Immune Subtypes and Landscape of Squamous Cell Carcinoma. Clin Cancer Res. 25 (12), 3528-3537 (2019).

32. Callari, M. et al. Subtype-Specific Metagene-Based Prediction of Outcome after Neoadjuvant and Adjuvant Treatment in Breast Cancer. Clin Cancer Res. 22 (2), 337-345 (2016).

33. George, S. et al. Loss of PTEN Is Associated with Resistance to Anti-PD-1 Checkpoint Blockade Therapy in Metastatic Uterine Leiomyosarcoma. Immunity. 2017;46(2):197-204. 
34. Burr, M. L. et al. CMTM6 maintains the expression of PD-L1 and regulates anti-tumour immunity. Nature. 549 (7670), 101-105 (2017).

35. Wang, L., Li, F., Sheng, J. \& Wong, S. T. A computational method for clinically relevant cancer stratification and driver mutation module discovery using personal genomics profiles. BMC Genomics. 7 (Suppl 7), S6 (2015).

36. Endris, V. et al. Measurement of tumor mutational burden (TMB) in routine molecular diagnostics: in silico and real-life analysis of three larger gene panels. Int J Cancer. 144 (9), 2303-2312 (2019).

37. Tran, E. et al. T-cell transfer therapy targeting mutant KRAS in cancer. N Engl J Med. 375 (23), 22552262 (2016).

38. Chowell, D. et al. Patient HLA class I genotype influences cancer response to checkpoint blockade immunotherapy. Science. 359 (6375), 582-587 (2018).

39. Xiang, X. et al. Prognostic value of PD-L1 expression in patients with primary solid tumors. Oncotarget. 9, 5058-5072 (2018).

40. Chifman, J., Pullikuth, A. \& Chou, J. W. Conservation of immune gene signatures in solid tumors and prognostic implications. BMC Cancer. 16 (1), 911 (2016).

41. Nirmal, A. J. et al. Immune cell gene signatures for profiling the microenvironment of solid tumors. Cancer Immunol Res. 6 (11), 1388-1400 (2018).

42. Sotiriou, C. et al. Gene expression profiling in breast cancer: understanding the molecular basis of histologic grade to improve prognosis. J Natl Cancer Inst. 98 (4), 262-272 (2006).

43. Camp, R. L., Dolled-Filhart, M. \& Rimm, D. L. X-tile: a new bio-informatics tool for biomarker assessment and outcome-based cut-point optimization. Clin Cancer Res. 10 (21), 7252-7259 (2004).

\section{Figures}


A

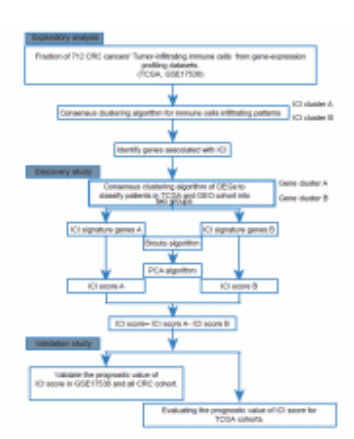

$E$

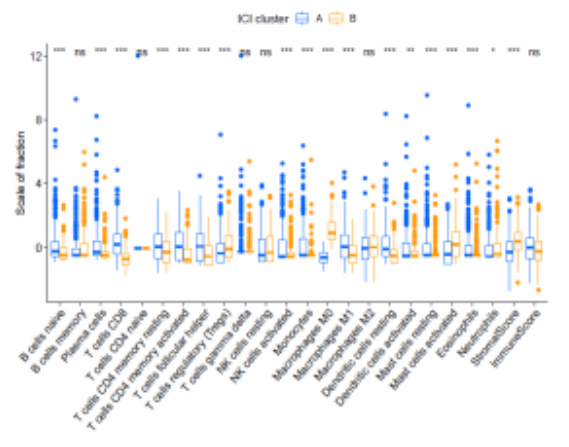

B

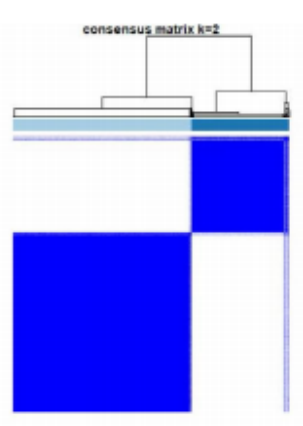

$\mathrm{F}$
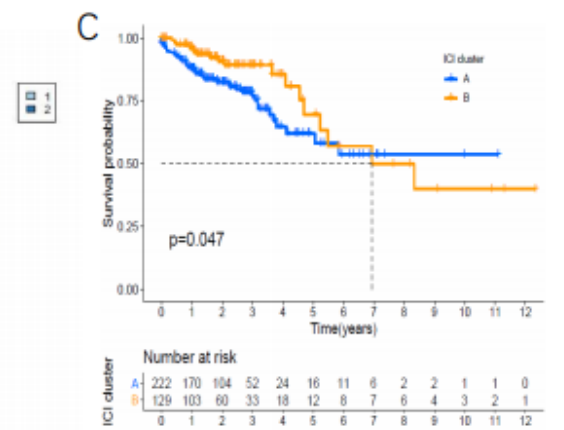

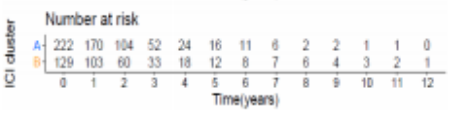

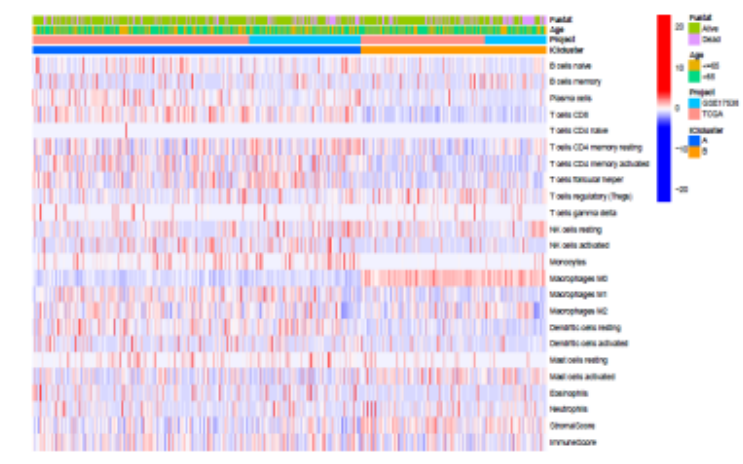

D

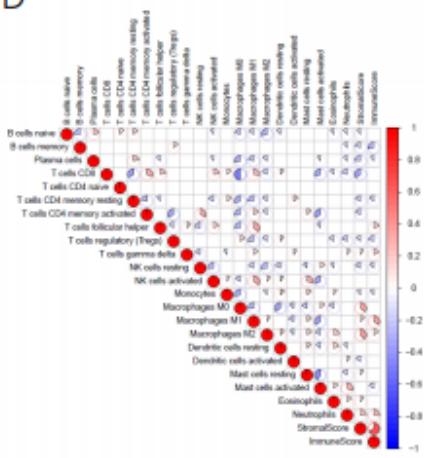

G

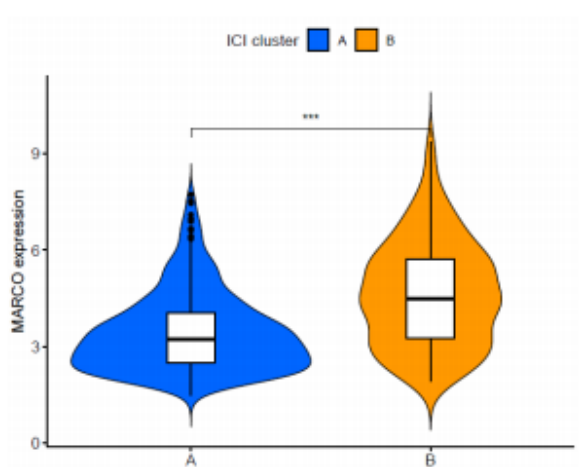

Figure 1

The TME-associated immune cell landscape of CRC (A) An overview of CRC sample selection. (B) Immune cell infiltration (ICI)-based CRC sample matrices. (C) CRC patient OS for individuals with different $\mathrm{ICl}$ subtypes was assessed using Kaplan-Meier curves and log-rank tests $(p=0.047)$. (D) Tumorinfiltrating cell interactions. (E) Relative frequencies of different tumor-infiltrating immune cell types in the two ICl clusters ( ${ }^{\star} \mathrm{p}<0.05 ;{ }^{* \star} \mathrm{p}<0.01 ; * \star \star p<0.001$; Wilcoxon test). (F) ICl populations in CRC cohorts were subjected to an unsupervised clustering analysis, with columns corresponding to samples and rows corresponding to tumor-infiltrating immune cells. (G) MARCO expression levels in different ICl clusters (Wilcoxon test, $\mathrm{p}<0.001$ ). 
A

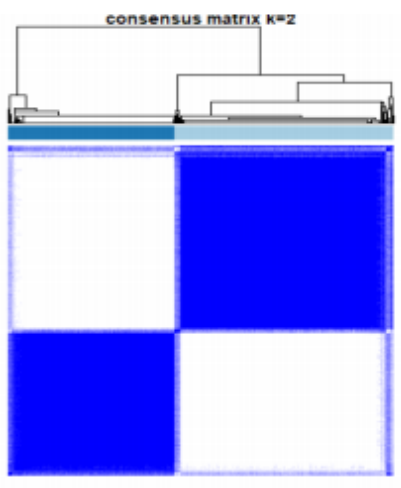

D

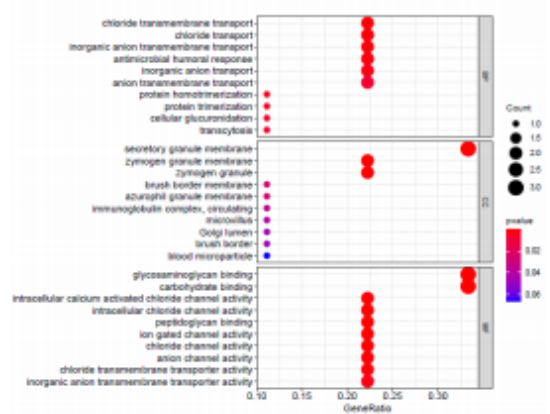

B

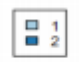

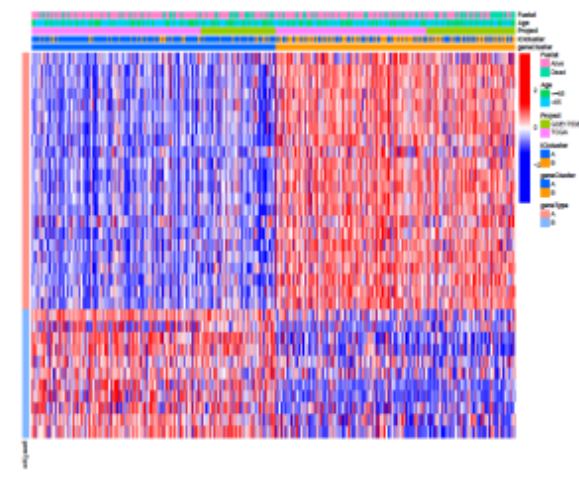

E

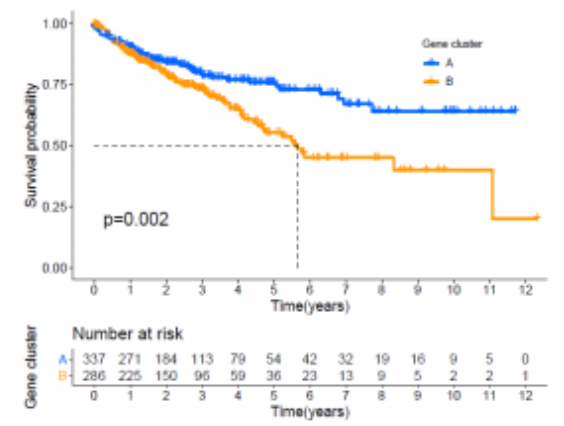

C

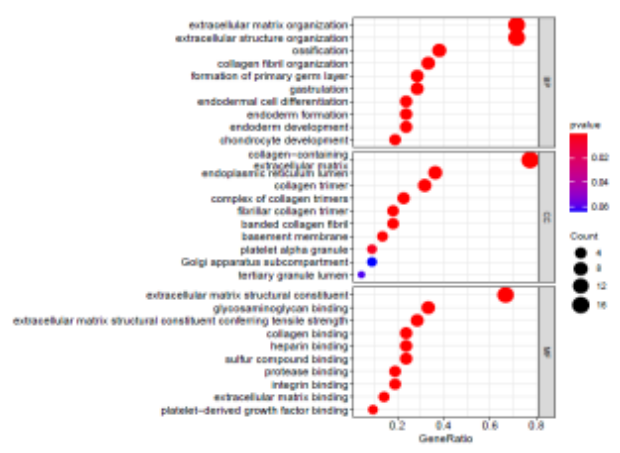

F

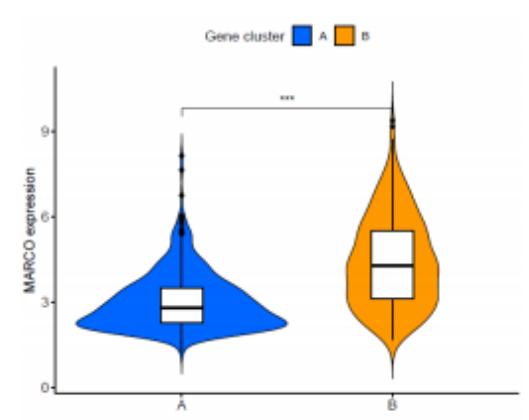

Figure 2

Immune Gene-Related Subtype Identification (A) Consensus matrices for CRC samples grouped according to DEG profiles. (B) Common DEGs between the two ICl cluster groups were subjected to unsupervised clustering to yield clusters $A$ and B. ( $C$ and D) Gene Ontology (GO) enrichment analyses of $\mathrm{ICl}$-related signature genes in clusters $A(C)$ and $B(D)$. Numbers of genes associated with the indicated GO terms are shown on the x-axis. (E) Survival outcomes were compared between patient groups with a Kaplan-Meier curve ( $p=0.002$; log-rank test). (F) MARCO expression levels in different ICI clustersWilcoxon test, $p<0.001)$. 


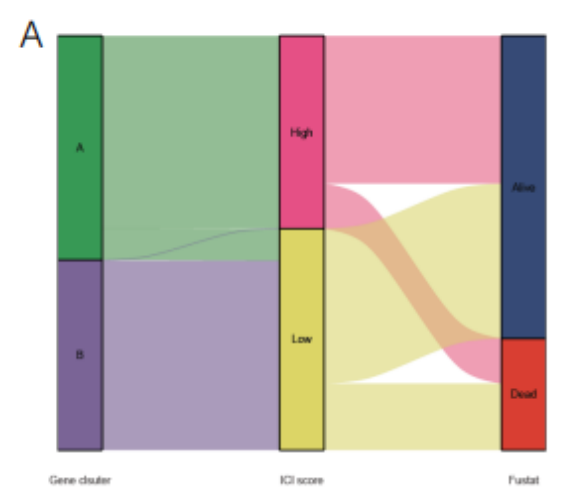

B

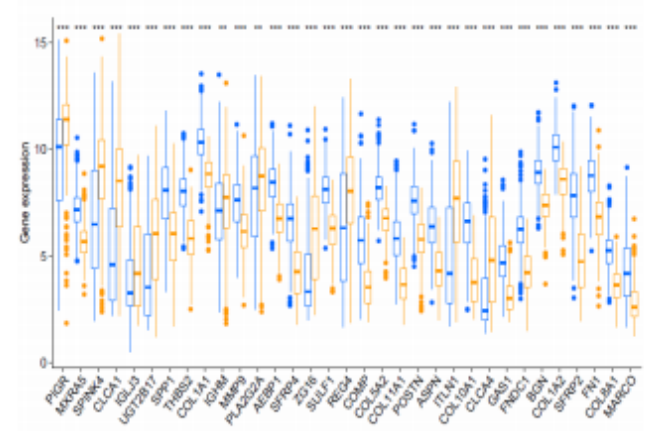

C

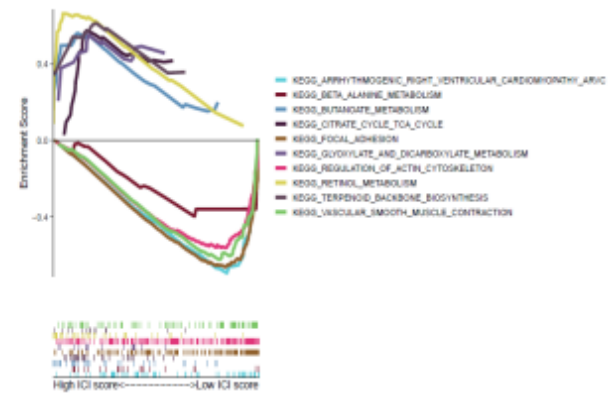

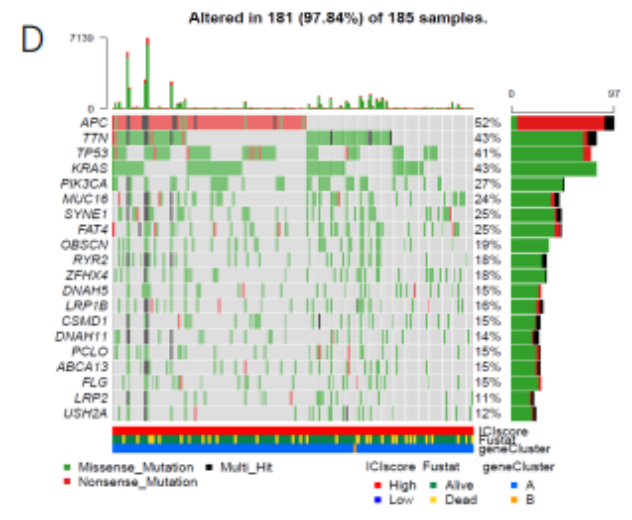

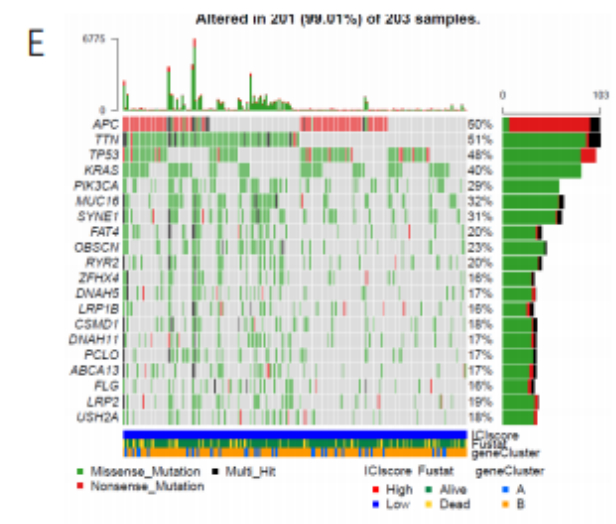

Figure 3

Correlations Between ICl Scores and Somatic Variants (A) ICl gene cluster distributions in groups with different ICl scores and survival outcomes are shown using an alluvial diagram. (B) Immune-checkpointrelated and immune activation-related genes present in low and high $\mathrm{ICl}$ score subgroups. (C) Enrichment plots demonstrating the enrichment of regulation- and vascular-related signaling pathways in the low $\mathrm{ICI}$ score subgroup, and butanoate- and retinol-related signaling pathways in the high ICI score subgroup. (D and E) High (red) and low (blue) ICl scores were used to construct oncoPrint, with columns corresponding to individual patients. 
A

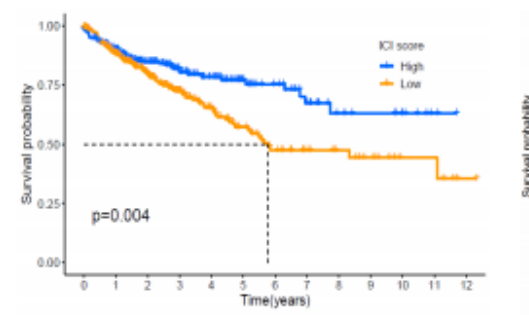

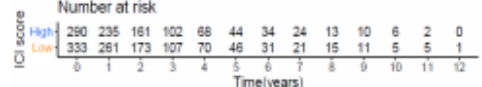

E

Patients with age $<=65$ ICl soore - High - Low

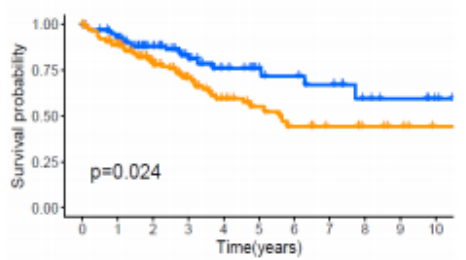

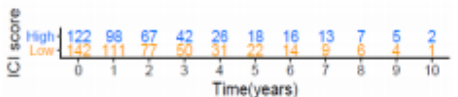

F
B

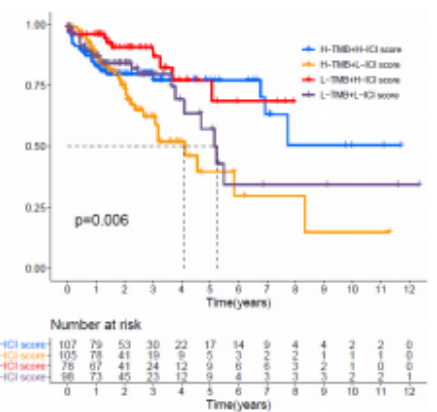

Patients with T3-4

ICl soore - High - Low

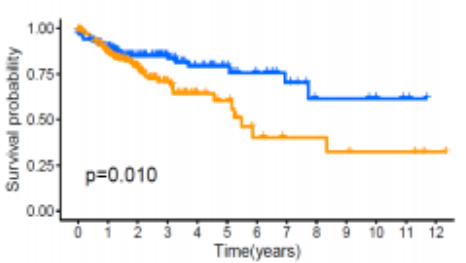

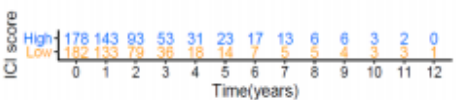

C

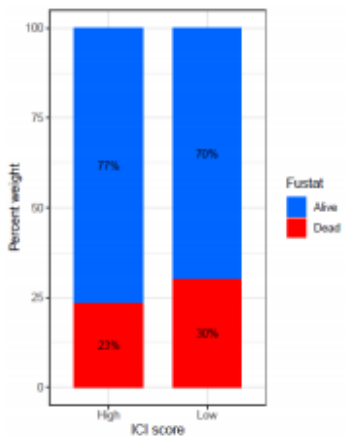

G

Patients with MALE

ICl score - High - Low

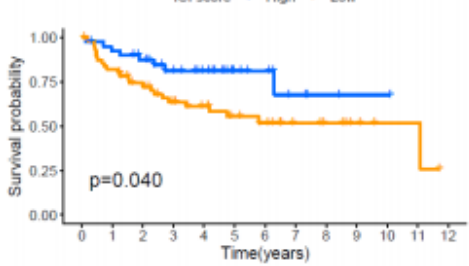

童
D

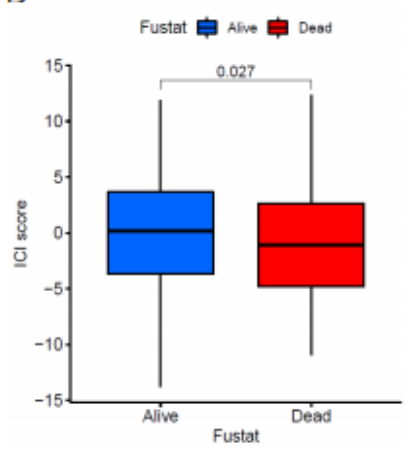

$\mathrm{H}$

Patients with FEMALE

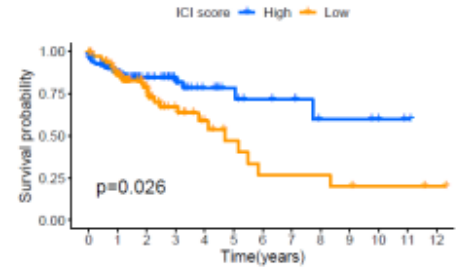

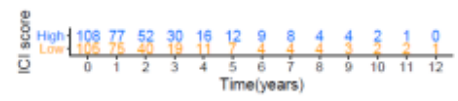

\section{Figure 4}

Evaluation of the prognostic value of $\mathrm{ICI}$ scores $(\mathrm{A})$ The survival outcomes of $\mathrm{CRC}$ patients in the low and high ICI score groups in the TCGA dataset were evaluated via Kaplan-Meier curves and log-rank tests $(p=0.004)$. (B) TCGA-CRC patients were stratified according to TMB and ICI scores, after which differences in OS outcomes among cohorts were analyzed ( $p=0.006$, log-rank test). (C) Survival outcomes and clinical responsiveness in those with low and high $\mathrm{ICl}$ scores. (D) Differences in ICl scores between surviving and non-surviving patients (Wilcoxon test, $p=0.027$ ). (E) Survival outcomes for patients $\leq 65-$ years-old with low and high ICI scores were evaluated ( $p=0.024$; log-rank test). ( $F$ ) Survival outcomes for CRC patients with advanced disease with low and high ICI scores were evaluated ( $p=0.01$; log-rank test). $(\mathrm{G}$ and $\mathrm{H})$ The relationship between survival and $\mathrm{ICI}$ scores in male or female patients was evaluated $(p=0.04$; log-rank test). 